\title{
Intra-Subject Fit Variability for Field Microphone- In-Real-Ear Attenuation Measurement for Custom Molded Earplugs
}

\author{
Jérémie Voix and Cécile Le Cocq \\ Département de génie mécanique, École de technologie supérieure, Université du Québec, Montréal, (QC), H3C \\ $1 K 3$, Canada
}

(Received 3 December 2009; accepted 21 April 2010)

\begin{abstract}
Over the last few years, several field attenuation measurement systems (FAMS) have been introduced to the industrial marketplace to measure the individual end-user attenuation for some hearing protection devices (HPDs). Although individual measurement is necessary to determine whether a given user is properly protected in his or her real-life noise environment (assuming that the exposure level is known), one unknown remains with a FAMS measurement: how reliable are the predictions made from the instantaneous measurement (over a few minutes), for determining the attenuation that will be achieved later in the field (over months or years) by the end-user who may fit them slightly differently every time? This paper will address that question for one FAMS, the field microphonein-real-ear (F-MIRE) measurement technique, and we will study, in the laboratory, how consistently subjects can fit and refit HPDs without assistance. A new metric, the intra-subject fit variability, will be introduced and will be quantified for custom-molded earplugs as fitted by inexperienced test subjects. This paper will present the experimental process used and statistical calculations performed to quantify the intra-subject fit variability. The number of successive refits required for a given prediction accuracy will also be presented, as well as the uncertainty component associated with the intra-subject fit variability when using an F-MIRE field attenuation measurement system.
\end{abstract}

\section{INTRODUCTION}

The goal of this study is to state how consistently a person can fit and refit a given HPD by himself or herself. Such a study is particularly important now that FAMS are commonly available to the hearing conservation community. Although FAMS may differ substantially in their methodology (a comprehensive list of FAMS available at the time of this writing exists ${ }^{1}$ ) they still have a common point: they only can take "snapshots" of the effective HPD attenuation. One could argue that the attenuation values reported by such FAMS are incomplete if no provision is made for how variable the fit of the HPD can be after the initial FAMS snapshot, hence the exact aim of the current study is to look at how consistently subjects can fit and refit HPDs without assistance. The FAMS that will be used in this study is the F-MIRE; it is currently only usable for insert HPDs (earplugs), but it was brought to the industry in 2001 and is one of the oldest and most documented objective FAMS. ${ }^{2-7}$

With the F-MIRE, the sound pressure levels in the ear canal (under the hearing protector) and those outside the HPD are simultaneously measured. Using suitable correction factors to account for known and quantifiable acoustic differences between the F-MIRE and REAT (real-ear attenuation at threshold), ${ }^{3,5}$ the values can be used to accurately estimate the HPD's attenuation. The F-MIRE system incorporates a dual-element miniature microphone and associated proprietary technology. One section of the dual-element microphone couples through the earplug to pick up the sound pressure levels in the ear canal, and the other section measures the external sound field. Broadband pink noise is presented through a small loudspeaker in front of the subject with proper equalization for the speaker fre-

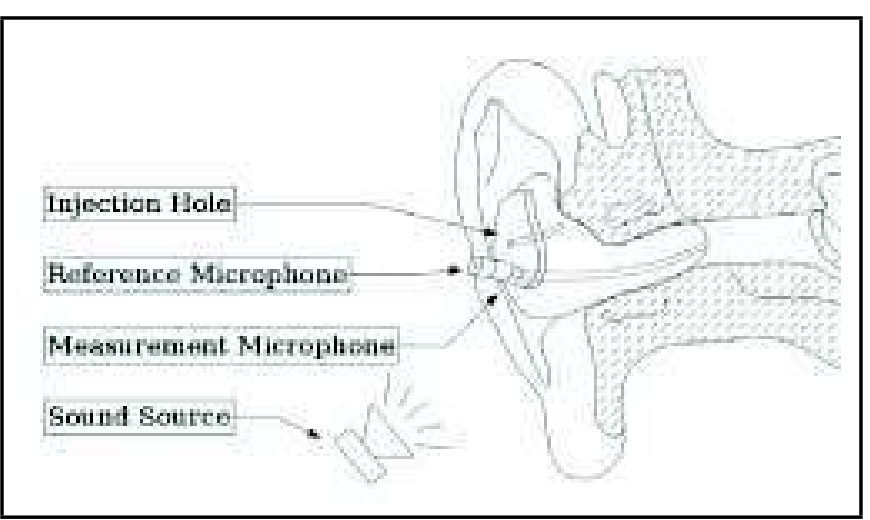

Figure 1. F-MIRE setup for an instrumented custom earplug with callouts for all important components.

quency response. The actual measurement takes about $10 \mathrm{sec}-$ onds for one fit in one ear for the standard seven test frequencies from $125 \mathrm{~Hz}$ to $8 \mathrm{kHz}$, from which an overall noise reduction rating or personal attenuation rating (PAR) is calculated. Though the PAR appears to be an exact number, it also contains its own variability, albeit much less than in the classical approach of using mean laboratory data to make individual field predictions. The extent of variability in PAR is defined and explicitly provided with the measurement, as it will be presented in section 3.2. F-MIRE is currently commercially available under two brand names, SonoPass ${ }^{\circledR}$ from Sonomax and E-A-Rfit ${ }^{\mathrm{TM}}$ from 3M/Aearo. The latter system has the ability to test both custom and disposable earplugs designed with a probe tube to measure the occluded signal. The SonoPass ${ }^{\circledR}$ system version 3.2 was used in this study. 